\title{
Trivial dynamics in discrete-time systems : carrying simplex and translation arcs
}

\author{
Niu, Lei
}

2018-06

Niu , L \& Ruiz-Herrera , A 2018 , ' Trivial dynamics in discrete-time systems : carrying

pÿsimplex and translation arcs ' , Nonlinearity , vol. 31 , no. 6 , pp. 26332650 . https://doi.org/10.1088/1361-6544/aa

http://hdl.handle.net/10138/301293

https://doi.org/10.1088/1361-6544/aab46e

acceptedVersion

Downloaded from Helda, University of Helsinki institutional repository.

This is an electronic reprint of the original article.

This reprint may differ from the original in pagination and typographic detail.

Please cite the original version. 


\title{
Trivial dynamics in discrete-time systems: carrying simplex and translation arcs
}

\author{
Lei $\mathrm{Niu}^{1}$ and Alfonso Ruiz-Herrera ${ }^{2}$ \\ ${ }^{1}$ Department of Mathematics and Statistics, FIN-00014 University of Helsinki, \\ Finland \\ 2 Departamento de Matematicas, Universidad de Oviedo, Oviedo, Spain \\ E-mail: lei.niu@helsinki.fi, ruizalfonso@uniovi.es
}

\begin{abstract}
In this paper we show that the dynamical behavior in $\mathbb{R}_{+}^{3}$ (first octant) of the classical Kolmogorov systems $T\left(x_{1}, x_{2}, x_{3}\right)=\left(x_{1} F_{1}(x), x_{2} F_{2}(x), x_{3} F_{3}(x)\right)$ of competitive type admitting a carrying simplex can be sometimes determined completely by the number of fixed points on the boundary and the local behavior around them. Roughly speaking, $T$ has trivial dynamics (i.e. the omega limit set of any orbit is a connected set contained in the set of fixed points) provided $T$ has exactly four hyperbolic nontrivial fixed points $\left\{p_{1}, p_{2}, p_{3}, p_{4}\right\}$ in $\partial \mathbb{R}_{+}^{3}$ with $\left\{p_{1}, p_{3}\right\}$ local attractors on the carrying simplex and $\left\{p_{2}, p_{4}\right\}$ local repellers on the carrying simplex; and there exists a unique hyperbolic fixed point in $\operatorname{Int} \mathbb{R}_{+}^{3}$. Our results are applied to some classical models including the Leslie-Gower models, Atkinson-Allen systems and Ricker maps.
\end{abstract}

AMS classification scheme numbers: 37Cxx, 37E30, 37N25, 92D25 


\section{Introduction}

For planar continuous flows, every non-empty compact omega limit set of an orbit containing no equilibria is a closed orbit by the Poincaré-Bendixson theorem. This theorem also applies to three-dimensional (3D) strongly continuous-time competitive systems due to Hirsch's carrying simplex theory [1]. Roughly speaking, this theory states that any strongly competitive system of differential equations of Kolmogorov type possesses a globally attracting codimension-one invariant hypersurface $\Sigma$ such that every nontrivial orbit is asymptotic to one in $\Sigma$. Therefore, the Poincaré-Bendixson theorem precludes any kind of chaos in these three dimensional systems and makes the dynamics easy to analyze [2, 3].

Based on the existence of a carrying simplex, Zeeman proposed in [4] a classification (the equivalence relation is called nullcline equivalence) for the Lotka-Volterra (LV) system

$$
\frac{d x_{i}}{d t}=x_{i}\left(r_{i}-\sum_{j=1}^{3} b_{i j} x_{j}\right), \quad i=1,2,3,
$$

where $r_{i}, b_{i j}$ are all positive real numbers. In these 33 equivalence classes, she further showed that every solution of (1) tends to some equilibrium when there is no interior equilibrium (classes $1-18$ ) or there is a unique interior equilibrium with index -1 (classes 19 - 25). Driessche and Zeeman [5] further ruled out closed orbits in classes 32 and 33, and hence these two classes also have trivial dynamics. Nontrivial dynamics (such as closed orbits and heteroclinic cycles) can only occur in classes $26-31$ which admit a unique interior equilibrium with index 1 . The reader can consult, for instance, [6, 7, 8], for additional results on Lotka-Volterra systems.

For discrete-time competitive systems, there have also been a lot of literature on the existence of a carrying simplex $\Sigma$ since the early work of Smith [9] and Hirsch [1]; see [10, 11, 12, 13, 14, 15, 16] for results on carrying simplices for discrete-time systems. Recently, Balreira, Elaydi and Luís provided in [17] a criterion on the global stability of discrete-time competitive systems via the carrying simplex; Mierczyński [18] proved that the carrying simplex for a three-dimensional competitive map is actually a $C^{1}$ submanifold-with-corners neatly embedded in the first octant under mild conditions. Just like the continuous-time competitive systems, in order to investigate the global dynamics of such discrete-time systems, it suffices to investigate the dynamics on $\Sigma$. Even so, only very few tools can apply to such discrete-time systems. In particular, the Poincaré-Bendixson theorem does not hold, because chaotic behavior can arise in twoor even one-dimensional discrete-time systems (such as the Hénon map [19] and the Ricker map [20]). Therefore, it is still a challenging topic to study the global dynamics of such discrete-time systems, even for low-dimensional systems.

In this paper, we study the dynamical behavior of 3D discrete-time Kolmogorov systems

$$
T(x)=\left(T_{1}(x), T_{2}(x), T_{3}(x)\right)=\left(x_{1} F_{1}(x), x_{2} F_{2}(x), x_{3} F_{3}(x)\right)
$$


which possess a carrying simplex $\Sigma$, where $F_{i}(x)>0$ for any $x \in \mathbb{R}_{+}^{3}, i=1,2,3$. Under mild conditions, Jiang and Niu [21] deduced a "Poincaré-Hopf-like" index formula for these systems. Specifically,

$$
\sum_{\theta \in \mathcal{F}_{v}} \operatorname{index}(T ; \theta)+2 \sum_{\theta \in \mathcal{F}_{s}} \operatorname{index}(T ; \theta)+4 \sum_{\theta \in \mathcal{F}_{p}} \operatorname{index}(T ; \theta)=1,
$$

where $\operatorname{index}(T ; \theta)$ denotes the index of $T$ at a fixed point $\theta$, and $\mathcal{F}_{v}\left(\mathcal{F}_{s}, \mathcal{F}_{p}\right)$ denotes the set of all nontrivial axial (planar, interior) fixed points. A direct application of (3) is that one can obtain the existence and index of interior fixed points by the boundary fixed points. Moreover, based on index formula (3), an equivalent classification theory (the equivalence relation is called equivalent relative to $\partial \Sigma$; here $\partial \Sigma=\Sigma \cap \partial \mathbb{R}_{+}^{3}$ ) for the classical 3D Leslie-Gower models and Atkinson-Allen systems was established in [16] and [21, 22], respectively. Such a classification theory has also been established for the 3D Ricker maps having a carrying simplex in [23]. The three kinds of discretetime systems all have 33 stable equivalence classes like Zeeman's classification for LV systems. Together with Ruiz-Herrera's exclusion criterion [14], it was proved that the classes possessing no interior fixed point (classes $1-18$ ) have trivial dynamics and the whole dynamics on $\Sigma$ for each of these classes was also presented further; see [16, 21, 22, 23]. Nontrivial dynamics (e.g. Neimark-Sacker bifurcations) may occur in other classes possessing a unique interior fixed point which has index 1. Although Neimark-Sacker bifurcations were ruled out in the classes possessing a unique interior fixed point with index -1 (classes $19-25$ ), whether they have trivial dynamics or not remains an open problem. Specifically, for class 19 for 3D Leslie-Gower models 16] (see also class 19 for 3D Atkinson-Allen systems [21, 22] and Ricker maps [23]), there are only five fixed points on $\Sigma$, say $s_{\{1\}}, s_{\{2\}}, s_{\{3\}}, v_{\{1\}}, q$, where $q$ is the unique interior fixed point. The local behavior of the equilibria is the following: $q$ is hyperbolic and has index $-1, s_{\{2\}}, s_{\{3\}}$ are hyperbolic local attractors and $s_{\{1\}}, v_{\{1\}}$ are hyperbolic local repellers lying on $\partial \Sigma$. The dynamics (partial) on $\Sigma$ is as shown in Figure 1. Unfortunately, whether this class has trivial dynamics or not, i.e. whether each orbit in this class will converge to a fixed point or not, is unknown.

Recall that for 3D continuous-time competitive systems (such as LV systems [4, 6] and Gompertz systems [24]) which admit a carrying simplex with the dynamics shown in Figure 1, it follows from Poincaré-Bendixson theorem immediately that every orbit tends to a fixed point and the whole dynamics on $\Sigma$ can also be obtained further; see Figure 2. Naturally, it is interesting whether such discrete-time systems have the similar dynamics as continuous-time systems or not.

The main tool of this paper consists of a topological result to guarantee trivial dynamics for orientation preserving homeomorphisms defined on a planar disk $D$. This result is deduced in Section 2 and could be perceived not only as a technique for analyzing (2) but have its own interest. In fact, an orientation preserving homeomorphism $f$ from a topological disk $D \subset \mathbb{R}^{k}$ (definitions will be given in Section 2) onto itself with a unique fixed point $q$ of index -1 in Int $D$ has trivial dynamics. The application of this abstract result to (2) leads to surprising properties: the number of equilibria on the 


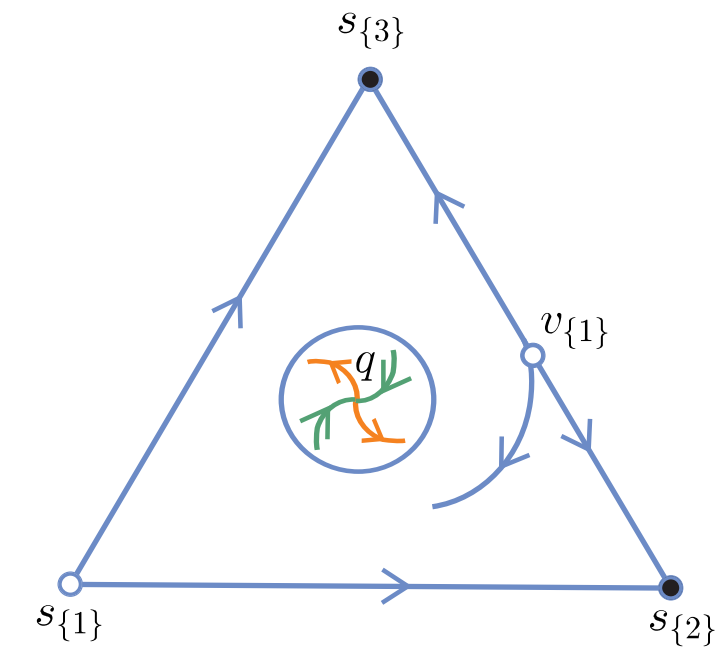

Figure 1: Partial dynamics on $\Sigma$ of class 19 for Leslie-Gower models, where the big circle $\bigcirc$ denotes a region of unknown dynamics. Redrawn from [16]. A fixed point is represented by a closed bullet $\bullet$ if it attracts on $\Sigma$, by an open bullet $\circ$ if it repels on $\Sigma$, and by the intersection of its hyperbolic manifolds if it is a saddle on $\Sigma$.

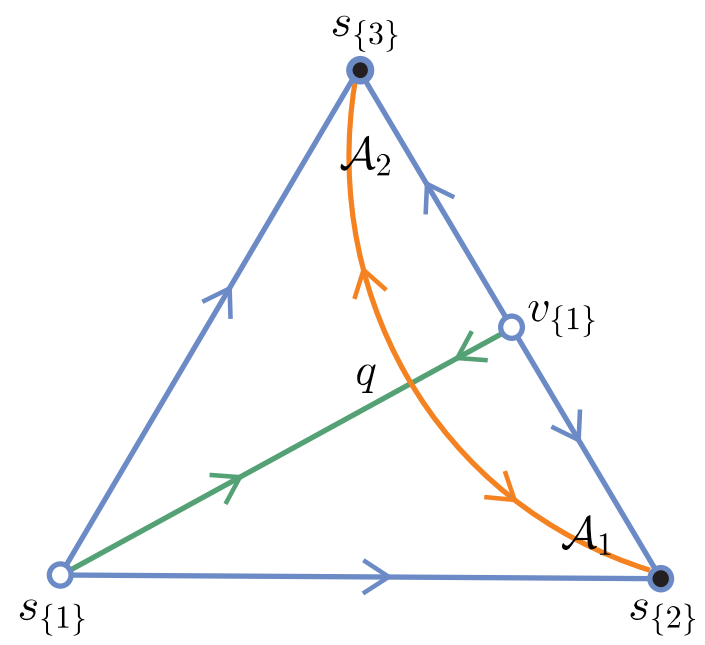

Figure 2: The whole dynamics on $\Sigma$ for continuous-time systems admitting a carrying simplex with the dynamics shown in Figure 1, where $\mathcal{A}_{1}$ (resp. $\mathcal{A}_{2}$ ) is the basin of attraction of $s_{\{2\}}\left(\operatorname{resp} . s_{\{3\}}\right)$ on $\Sigma$. The fixed point notation is as in Figure 1 .

boundary and the local behavior around them can determine completely the dynamical behavior of the system when there is a unique positive fixed point which has index -1 . Specifically, if $T$ has exactly four hyperbolic fixed points $\left\{p_{1}, p_{2}, p_{3}, p_{4}\right\}$ on the boundary of $\Sigma$ with $\left\{p_{1}, p_{3}\right\}$ local attractors and $\left\{p_{2}, p_{4}\right\}$ local repellers; and there exists a unique hyperbolic fixed point in the interior of $\Sigma$, then $T$ has trivial dynamics. We will apply the results to some classical models such as the Leslie-Gower models, the Atkinson-Allen systems, and the Ricker maps. As a consequence, we also solve some corresponding open problems presented in [16, 21, 22, 23, i.e. these systems (3D) do have trivial dynamics 
when they admit a unique positive fixed point having index -1 , and hence the stable classes $19-25$ for these systems have trivial dynamics. In particular, the discrete-time systems admitting a carrying simplex with the dynamics shown in Figure 1 have the similar dynamics as continuous-time systems which is as shown in Figure 2.

\section{Main Topological Results}

The aim of this section is to give a criterion of trivial dynamics for orientation preserving homeomorphisms defined on a topological disk $D$ with a unique fixed point in $\operatorname{Int} D$, the interior of $D$. As usual, a topological disk is a set contained in $\mathbb{R}^{k}(k \geq 2)$ which is homeomorphic to the closed unit disk $\overline{\mathcal{D}_{1}}=\left\{(x, y) \in \mathbb{R}^{2}: x^{2}+y^{2} \leq 1\right\}$, where $\mathbb{R}^{k}$ denotes the $k$-dimensional Euclidean space. A striking consequence of our result is that the whole dynamics of those maps can be determined from the knowledge of the number of fixed points in the boundary of $D$ and the local behavior around them. For a map $f: D \mapsto D$, we denote by Fix $(f)$ the set of all its fixed points, by $\omega(x, f)$ the omega limit set of the orbit emanating from $x$, and by index $(f ; q)$ the topological index of $f$ at $q$; see 25,26 .

- Throughout this section $D$ denotes a topological disk, and $f$ is an orientation preserving homeomorphism from $D$ onto $D$.

Next we state the main theorem of this section.

Theorem 2.1. Assume that Fix $(f) \cap \operatorname{Int} D=\{q\}$ and index $(f ; q)=-1$. Then $f$ has trivial dynamics, that is, for all $x \in D, \omega(x, f)$ is a connected set contained in Fix $(f)$. Furthermore, if $f$ has only finitely many fixed points, then any orbit of $f$ converges to some fixed point.

\subsection{Translation arcs and proof of Theorem 2.1}

The proof of Theorem 2.1 relies on the theory of translation arcs. For the reader's convenience, we recall some known results, (see [27, 28, 29, 30] for a more detailed discussion on this technique).

A translation arc $\alpha$ for an orientation preserving homeomorphism $h: D \mapsto D$ is an arc contained in $\operatorname{Int} D$ with ends at $q$ and $h(q)$ and satisfying that

$$
h(\alpha \backslash\{h(q)\}) \cap(\alpha \backslash\{h(q)\})=\emptyset .
$$

First we collect the main two ingredients of the proof of Theorem 2.1.

Lemma 2.2 (Proposition 2.1 in [29]). Assume that $h: D \mapsto D$ is an orientation preserving homeomorphism and $D_{1} \subseteq \operatorname{Int} D$ is a topological disk with

$$
h\left(D_{1}\right) \cap D_{1}=\emptyset .
$$


Then, given points $z_{1}, \ldots, z_{n} \in \operatorname{Int} D_{1}$, there is a translation arc $\alpha$ with ends at $q, h(q) \in \operatorname{Int} D$ and satisfying that

$$
z_{1}, \ldots, z_{n} \in \alpha \backslash\{q, h(q)\}
$$

Lemma 2.3 (Brouwer's lemma [30]). Assume that $h: D \mapsto D$ is an orientation preserving homeomorphism and $\alpha$ is a translation arc with

$$
h^{n_{0}}(\alpha) \cap \alpha \neq \emptyset
$$

for some $n_{0} \geq 2$. Then there is a Jordan curve $\Gamma \subset \operatorname{Int} D \backslash F i x(h)$ such that

$$
\operatorname{deg}\left(i d-h, R_{i}(\Gamma)\right)=1
$$

with $R_{i}(\Gamma)$ the bounded connected component of $\mathbb{R}^{2} \backslash \Gamma$.

Proof of Theorem 2.1.

All the notions involved in the theorem are invariant by topological conjugacy. Therefore, it is not restrictive to assume that $D=\left\{(x, y): x^{2}+y^{2} \leq 1\right\}$.

Noticing that $f(\operatorname{Int} D)=\operatorname{Int} D$ and $f(\partial D)=\partial D$, we first show that for all $x \in \operatorname{Int} D$, $\omega(x, f) \subseteq F i x(f)$. Assume by contradiction that there is some $p \in \operatorname{Int} D$ such that

$$
\omega(p, f) \nsubseteq F i x(f) .
$$

Then there is a sequence $\sigma(n): \mathbb{N} \mapsto \mathbb{N}$ strictly increasing so that

$$
f^{\sigma(n)}(p) \rightarrow r \in D
$$

with $f(r) \neq r$. Using that $f(\operatorname{Int} D)=\operatorname{Int} D$, we have that $f^{n}(p) \in \operatorname{Int} D$ for all $n \in \mathbb{N}$. Now, we take $\varepsilon>0$ such that the Euclidean closed ball centered at $r$ with radius $\varepsilon$, say $D_{1}$, which satisfies that

$$
f\left(D \cap D_{1}\right) \cap\left(D \cap D_{1}\right)=\emptyset .
$$

Note that $f(r) \neq r$. Since $f^{\sigma(n)}(p) \rightarrow r$, there are two indices $k_{1}<k_{2}$ such that $\sigma\left(k_{2}\right)-\sigma\left(k_{1}\right)>2$ and $f^{\sigma\left(k_{1}\right)}(p), f^{\sigma\left(k_{2}\right)}(p) \in \operatorname{Int}\left(D \cap D_{1}\right)$. Next, take $\tilde{D}_{1}$ a topological disk contained in $\operatorname{Int}\left(D_{1} \cap D\right)$ so that $f^{\sigma\left(k_{1}\right)}(p), f^{\sigma\left(k_{2}\right)}(p) \in \operatorname{Int}\left(\tilde{D}_{1}\right)$. This topological disk can be constructed as follows. Take $\gamma$ an arc joining $f^{\sigma\left(k_{1}\right)}(p), f^{\sigma\left(k_{2}\right)}(p)$ contained in $\operatorname{Int}\left(D_{1} \cap D\right)$ and set $\tilde{D}_{1}=\{(x, y) \in D: \operatorname{dist}((x, y), \gamma) \leq \delta\}$ with $\delta>0$ small enough; see Figure 3 .

Note that $f\left(\tilde{D}_{1}\right) \cap \tilde{D}_{1}=\emptyset$. Now, we can apply Lemma 2.2 to obtain a translation arc $\alpha$ such that $f^{\sigma\left(k_{1}\right)}(p), f^{\sigma\left(k_{2}\right)}(p) \in \alpha$. Observe that

$$
f^{\sigma\left(k_{2}\right)}(p) \in \alpha \cap f^{\sigma\left(k_{2}\right)-\sigma\left(k_{1}\right)}(\alpha) .
$$

Then, by Lemma 2.3, there is a Jordan curve $\Gamma \subset \operatorname{Int} D \backslash\{q\}$ so that $\operatorname{deg}\left(i d-f, R_{i}(\Gamma)\right)=$ 1. This is a contradiction because the uniqueness of the fixed point $q$ and the condition index $(f ; q)=-1$ imply that $\operatorname{deg}\left(i d-f, R_{i}(\Gamma)\right)=-1$ if $q \in R_{i}(\Gamma)$ and 0 otherwise. At 


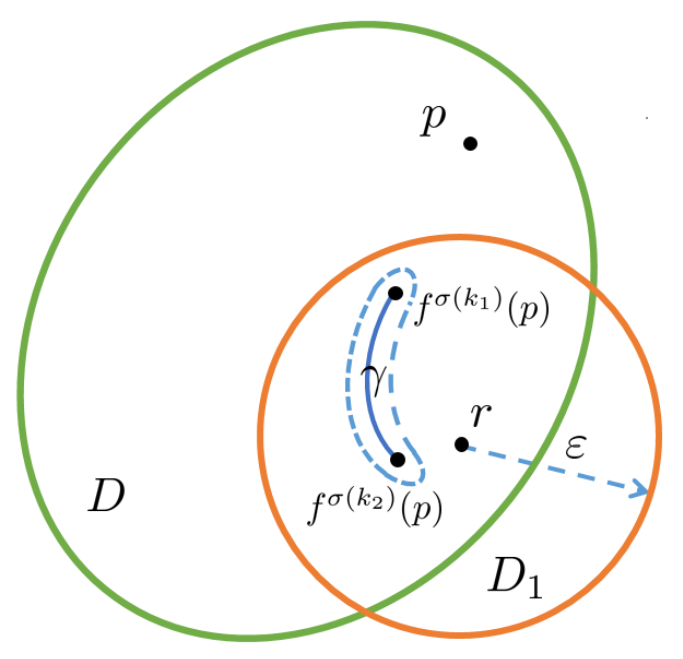

Figure 3: The construction of $\tilde{D}_{1}$.

this moment we have proved that $\omega(x, f) \subseteq F i x(f)$ for all $x \in \operatorname{Int} D$.

Now suppose that there is some $z \in \partial D$ such that $\omega(z, f) \nsubseteq F i x(f)$. Also, there is a strictly increasing sequence $\sigma(n): \mathbb{N} \mapsto \mathbb{N}$ so that

$$
f^{\sigma(n)}(z) \rightarrow r \in D
$$

with $f(r) \neq r$. Take an Euclidean closed ball $D_{1}$ centered at $r$ satisfying

$$
f\left(D \cap D_{1}\right) \cap\left(D \cap D_{1}\right)=\emptyset .
$$

Then there are two indices $k_{1}<k_{2}$ with $\sigma\left(k_{2}\right)-\sigma\left(k_{1}\right)>2$ such that $f^{\sigma\left(k_{1}\right)}(z)$, $f^{\sigma\left(k_{2}\right)}(z) \in D \cap D_{1}$. By continuity of $f^{\sigma\left(k_{1}\right)}$ and $f^{\sigma\left(k_{2}\right)}$, there is a neighborhood $U_{z} \subset D$ of $z$ satisfying that $f^{\sigma\left(k_{1}\right)}\left(U_{z}\right), f^{\sigma\left(k_{2}\right)}\left(U_{z}\right) \subseteq D \cap D_{1}$. Choose $p \in U_{z} \cap \operatorname{Int} D$. Therefore, one has $f^{\sigma\left(k_{1}\right)}(p), f^{\sigma\left(k_{2}\right)}(p) \in \operatorname{Int}\left(D \cap D_{1}\right)$. Then repeat the above arguments for the case $p \in \operatorname{Int} D$, one will obtain the contradiction. Thus, we have proved that $\omega(x, f) \subseteq F i x(f)$ for all $x \in D$.

We now show that $\omega(x, f)$ is a connected set. By a contradiction suppose that $\omega(x, f)$ can be divided into two disjoint nonempty closed sets $\Omega_{1}$ and $\Omega_{2}$. Note that $\Omega_{i} \subset F i x(f)$, $i=1,2$, so $\Omega_{i}$ is invariant under $f$. While it is a contradiction because $\omega(x, f)$ is invariantly connected by [31, Theorem 5.2], i.e. $\omega(x, f)$ cannot be the union of two nonempty disjoint closed invariant sets. The last conclusion is immediate.

\subsection{Whole dynamics on $D$}

The aim of this subsection is to provide some dynamical insights of $f$ when it has exactly two locally asymptotically stable attractors and two locally asymptotically repellers. We will say that $p$ is locally asymptotically stable attractor if there exists $\left\{U_{n}\right\}$ a decreasing sequence of neighbourhoods of $p$ such that $f\left(U_{n}\right) \subset U_{n+1}$ with $\cap_{n=1}^{\infty} U_{n}=\{p\}$. The basin of attraction of $p$ is defined as

$$
\left\{r \in D: f^{n}(r) \longrightarrow p\right\}
$$


In an analogous manner, we can define a locally asymptotically repeller and the basin of repulsion simply replacing $f$ by $f^{-1}$. For the point $q$, that is essentially a saddle point, we define its stable manifold

$$
W^{s}(q, f)=\left\{y \in D: \lim _{n \rightarrow+\infty} f^{n}(y)=q\right\}
$$

and its unstable manifold

$$
W^{u}(q, f)=\left\{y \in D: \lim _{n \rightarrow+\infty} f^{-n}(y)=q\right\}
$$

Theorem 2.4. Assume that

- $F i x(f) \cap \partial D=\left\{p_{1}, p_{2}, p_{3}, p_{4}\right\}$;

- $p_{1}, p_{3}$ are local attractors and $p_{2}, p_{4}$ are local repellers;

- Fix $(f) \cap \operatorname{Int} D=\{q\}$, index $(f ; q)=-1$ and $\operatorname{index}\left(f^{-1} ; q\right)=-1$.

Then

(i) $\operatorname{Int} D=W^{s}(q, f) \cup\left(\mathcal{C}_{1} \cup \mathcal{C}_{3}\right) \cap \operatorname{Int} D$ where $\mathcal{C}_{1}$ (resp. $\left.\mathcal{C}_{3}\right)$ is the basin of attraction of $p_{1}$ (resp. $\left.p_{3}\right)$. Moreover, $\left\{q, p_{2}, p_{4}\right\} \subseteq \partial C_{1} \cap \partial C_{3}$;

(ii) Int $D=W^{u}(q, f) \cup\left(\mathcal{C}_{2} \cup \mathcal{C}_{4}\right) \cap \operatorname{Int} D$ where $\mathcal{C}_{2}$ (resp. $\left.\mathcal{C}_{4}\right)$ is the basin of repulsion of $p_{2}$ (resp. $p_{4}$ ). Moreover, $\left\{q, p_{1}, p_{3}\right\} \subseteq \partial C_{2} \cap \partial C_{4}$;

(iii) $W^{s}(q, f) \cup W^{u}(q, f) \cup\left\{p_{1}, p_{2}, p_{3}, p_{4}\right\}$ partitions $D$ into four invariant components, namely, $\mathcal{C}_{1} \cap \mathcal{C}_{2}, \mathcal{C}_{1} \cap \mathcal{C}_{4}, \mathcal{C}_{3} \cap \mathcal{C}_{1}$, and $\mathcal{C}_{3} \cap \mathcal{C}_{2}$; see Figures 4.)

Proof. By Theorem 2.1, we know that $f$ and $f^{-1}$ have trivial dynamics.

(i) Take $r \in \operatorname{Int} D$ with $r \notin C_{1} \cup C_{3}$. Since $f$ has trivial dynamics and the number of fixed points is finite, the sequence $\left\{f^{n}(r)\right\}$ converges to a fixed point. Such a fixed point has to be $q$ because $p_{2}, p_{4}$ are repellers and $r$ is not in the basin of attraction of $p_{1}$ or $p_{3}$. This claim proves that $\operatorname{Int} D=W^{s}(q, f) \cup\left(\mathcal{C}_{1} \cup \mathcal{C}_{3}\right) \cap \operatorname{Int} D$. It is clear that the boundary of the basin of attraction of $p_{1}$ or $p_{3}$ on the boundary of $D$ are the points $p_{2}$ and $p_{4}$. Finally, we take a point $r \in \operatorname{Int} D \cap \partial C_{1}$. It is clear that $r \notin C_{3}$ because $C_{3}$ is an open set and $C_{1} \cap C_{3}=\emptyset$. Then, arguing as above,

$$
f^{n}(r) \longrightarrow q
$$

Observe that $\partial C_{1}$ is an invariant set and so $q \in \partial C_{1}$. In the same way, we can prove that $q \in \partial C_{3}$.

(ii) The argument is the same as (i) simply using that $f^{-1}$ has trivial dynamics.

(iii) Since $f$ is homeomorphism, $W^{s}(q, f) \cup W^{u}(q, f)$ are invariant and $f(\partial D)=\partial D$, the conclusion is immediate.

The previous result does not use any hyperbolicity or smoothness conditions of $f$ and $q$. Therefore, it can be applied for non-smooth two dimensional manifolds. On the other hand, under the hypothesis of the theorem, we can not guarantee, for instance, 

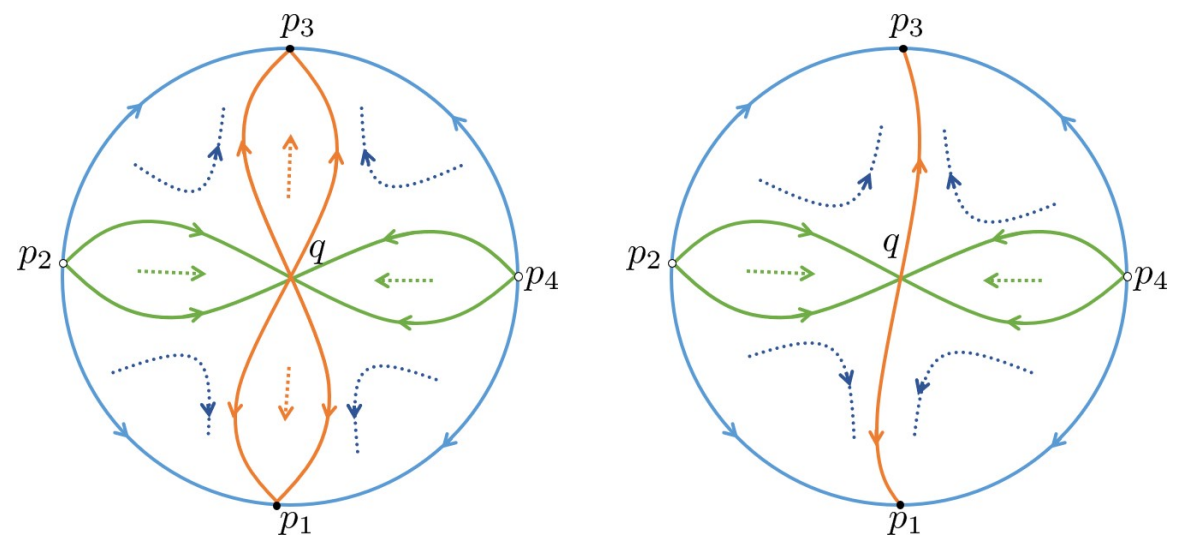

(a) $\partial C_{1}, \partial C_{3} \neq W^{s}(q, f) \cup\left\{p_{2}, p_{4}\right\}$, $\partial C_{2}, \partial C_{4} \neq W^{u}(q, f) \cup\left\{p_{1}, p_{3}\right\}$

(b) $\partial C_{1}, \partial C_{3} \neq W^{s}(q, f) \cup\left\{p_{2}, p_{4}\right\}$,

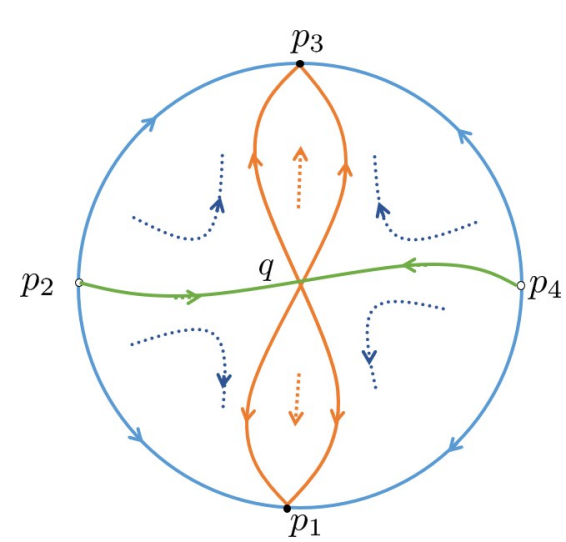

$\partial C_{2}=\partial C_{4}=W^{u}(q, f) \cup\left\{p_{1}, p_{3}\right\}$

(c) $\partial C_{1}=\partial C_{3}=W^{s}(q, f) \cup\left\{p_{2}, p_{4}\right\}$, $\partial C_{2}, \partial C_{4} \neq W^{u}(q, f) \cup\left\{p_{1}, p_{3}\right\}$

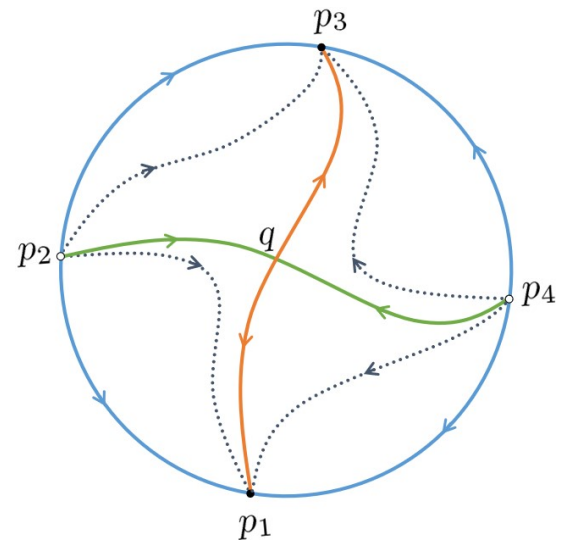

(d) $\partial C_{1}=\partial C_{3}=W^{s}(q, f) \cup\left\{p_{2}, p_{4}\right\}$,

$\partial C_{2}=\partial C_{4}=W^{u}(q, f) \cup\left\{p_{1}, p_{3}\right\}$

Figure 4: $W^{s}(q, f) \cup W^{u}(q, f) \cup\left\{p_{1}, p_{2}, p_{3}, p_{4}\right\}$ partitions $D$ into four invariant components.

that $\partial C_{1}=\partial C_{3}=W^{s}(q, f) \cup\left\{p_{2}, p_{4}\right\}$; see Figure 4(a)-(b). For case (d) in Figure 4 . $W^{s}(q, f) \cup\left\{p_{2}, p_{4}\right\}$ is a separatrix arc partitioning $D$ into two connected components $\mathcal{C}_{1}$ and $\mathcal{C}_{3}$, and $W^{u}(q, f) \cup\left\{p_{1}, p_{3}\right\}$ is also a separatrix arc partitioning $D$ into two connected components $\mathcal{C}_{2}$ and $\mathcal{C}_{4}$.

\section{Carrying Simplex vs Theorem 2.1}

The motivation of the abstract results of the previous section is to study discrete-time systems that describe the interaction of three competitive species. Specifically, we study $T: \mathbb{R}_{+}^{k} \mapsto \mathbb{R}_{+}^{k}$ of the type

$$
T(x)=\left(T_{1}(x), \cdots, T_{k}(x)\right)=\left(x_{1} F_{1}(x), \cdots, x_{k} F_{k}(x)\right),
$$

where $\mathbb{R}_{+}^{k}$ is the usual nonnegative cone in $\mathbb{R}^{k}$, and $F_{i}(x)>0$ for any $x \in \mathbb{R}_{+}^{k}$, $i=1, \cdots, k$. We link Theorem 2.1 with (4) via the carrying simplex, i.e. a codimensionone invariant manifold that determines completely the dynamical behavior of most 
competitive systems. In more detail, we say that $T$ admits a carrying simplex, denoted by $\Sigma$, if there is a subset of $\mathbb{R}_{+}^{k} \backslash\{0\}$ so that

(C1) $\Sigma$ is compact, invariant and unordered;

(C2) $\Sigma$ is homeomorphic via radial projection to the $(k-1)$-dimensional standard probability simplex $S^{k-1}=\left\{x \in \mathbb{R}_{+}^{k}: \sum_{i} x_{i}=1\right\}$;

(C3) for any $x \in \mathbb{R}_{+}^{k} \backslash\{0\}$, there is some $z \in \Sigma$ such that $\lim _{n \rightarrow \infty}\left|T^{n}(x)-T^{n}(z)\right|=0$;

(C4) $T(\Sigma)=\Sigma$, and $T: \Sigma \mapsto \Sigma$ is a homeomorphism.

The key advantage of the existence of a carrying simplex is that the dimension of the system is reduced. Consequently, we can apply, for instance, results of planar maps in 3D systems. We denote the interior of $\Sigma$, i.e. $\Sigma \cap \operatorname{Int} \mathbb{R}_{+}^{k}$ by $\operatorname{Int} \Sigma$, and the boundary of $\Sigma$, i.e. $\Sigma \cap \partial \mathbb{R}_{+}^{k}$ by $\partial \Sigma$.

Theorem 3.1. Let $T=\left(x_{1} F_{1}(x), x_{2} F_{2}(x), x_{3} F_{3}(x)\right): \mathbb{R}_{+}^{3} \mapsto \mathbb{R}_{+}^{3}$ be a $C^{1}$-map satisfying:

A1) $\partial F_{i}(x) / \partial x_{j}<0$ holds for any $x \in \mathbb{R}_{+}^{3}$ and $i, j=1,2,3$;

A2) $\left.T\right|_{\mathbb{H}_{\{i\}}^{+}}: \mathbb{H}_{\{i\}}^{+} \mapsto \mathbb{H}_{\{i\}}^{+}$has a fixed point $v_{\{i\}}=v_{i} e_{\{i\}}$ with $v_{i}>0$, $i=1,2,3$, where $\left.T\right|_{\mathbb{H}_{\{i\}}^{+}}$denotes the restriction of $T$ on $\mathbb{H}_{\{i\}}^{+}$(the ith positive coordinate axis), and $e_{\{i\}}$ is the unit vector pointing in the ith direction;

A3) $\forall x \in[0, v] \backslash\{0\}, F_{i}(x)+\sum_{j \in \kappa(x)} x_{j} \frac{\partial F_{i}(x)}{\partial x_{j}}>0$ holds for any $i \in \kappa(x)$ (or $F_{i}(x)+\sum_{j \in \kappa(x)} x_{i} \frac{\partial F_{i}(x)}{\partial x_{j}}>0$ holds for any $\left.i \in \kappa(x)\right)$, where $\kappa(x)=\left\{j: x_{j}>0\right\}$ is the support of $x, v=\sum v_{\{i\}}=\left(v_{1}, v_{2}, v_{3}\right)$ and the closed order interval $[0, v]=\left\{x \in \mathbb{R}_{+}^{3}: 0 \leq x_{i} \leq v_{i}, i=1,2,3\right\}$.

If $T$ has a unique fixed point $q \in \operatorname{Int} \mathbb{R}_{+}^{3}$ so that the eigenvalues of the Jacobian matrix at $q$ are $\lambda_{1}, \lambda_{2}, \lambda_{3}$ with $0<\lambda_{1} \leq \lambda_{2}<1<\lambda_{3}$, then the omega limit set of any orbit of $T$ is a connected set contained in Fix $(T)$; moreover, if $T$ has only finitely many fixed points, then any nontrivial orbit of $T$ tends to some nontrivial fixed point.

Proof. First, conditions A1)-A3) guarantee the existence of a carrying simplex $\Sigma$ by [16, Theorem 3.1]. For convenience, we denote by $\left.T\right|_{\Sigma}$ the restriction of $T$ to the carrying simplex. Now we show that $\operatorname{index}\left(\left.T\right|_{\Sigma} ; q\right)=-1$. Since 1 is not an eigenvalue of the Jacobian matrix of $T$ at $q, i d-T$ is a diffeomorphism in a neighbourhood of $q$. This implies that there is $\varepsilon_{0}>0$ so that the sphere $S_{\varepsilon} \subset \mathbb{R}^{3}$ which is centered at $q$ with radius $0<\varepsilon<\varepsilon_{0}$ satisfies that any closed and simple curve $\gamma \subset S_{\varepsilon}$ surrounding $q, \gamma-T(\gamma)$ is also a closed and simple curve surrounding the origin. Moreover, the local unstable manifold of $q$ intersects $S_{\varepsilon}$ at exactly two points. On the other hand, we know that the carrying simplex is a two dimensional invariant manifold (generally a non-smooth manifold) that attracts all non-trivial orbits of the system. By this remark, it is clear that, on the carrying simplex, $q$ is neither an attractor nor a repeller. In addition, since the unstable manifold of $q$ is uniquely defined, we can conclude that it is contained in the carrying simplex. In particular, the local unstable manifold of $q$ is contained in the carrying simplex. Now, we have all the ingredients to conclude that $i n \operatorname{dex}\left(\left.T\right|_{\Sigma} ; q\right)=-1$. 
To check this property, we apply the linkage between the degree of a planar map and the winding number. Given $S_{\varepsilon}$ a small sphere centered at $q, \Sigma \cap S_{\varepsilon}$ is a Jordan curve $\gamma_{\varepsilon}$ in the carrying simplex. By the previous discussion, $\beta_{\varepsilon}=\gamma_{\varepsilon}-T_{\Sigma}\left(\gamma_{\varepsilon}\right)$ is a simple and closed curve surrounding 0 . Therefore, the winding number of $\beta_{\varepsilon}$ is 1 or -1 . We now discard the winding number 1 . Denote by $R_{i}\left(\gamma_{\varepsilon}\right)$ and $R_{e}\left(\gamma_{\varepsilon}\right)$ the interior and the exterior domains limited by $\gamma_{\varepsilon}$ in the carrying simplex respectively. Since the local unstable manifold of $q$ is contained in the carrying simplex, we deduce that $W_{l o c}^{u}(q) \cap \gamma_{\varepsilon}=\left\{r_{1, \varepsilon}, r_{2, \varepsilon}\right\}$ for all $0<\varepsilon<\varepsilon_{0}$. Thus, $T\left(r_{1, \varepsilon}\right), T\left(r_{2, \varepsilon}\right) \in R_{e}\left(\gamma_{\varepsilon}\right)$. On the other hand, the points $r_{1, \varepsilon}$ and $r_{2, \varepsilon}$ split the curve $\gamma_{\varepsilon}$ into two arcs, $\alpha_{1, \varepsilon}$ and $\alpha_{2, \varepsilon}$. In these two arcs, there exist

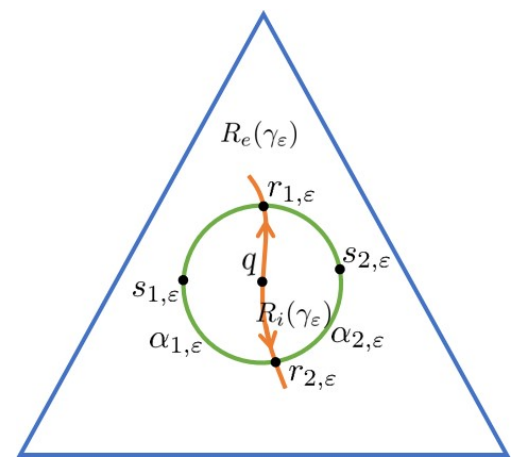

Figure 5: $r_{1, \varepsilon}$ and $r_{2, \varepsilon}$ split the curve $\gamma_{\varepsilon}$ into two arcs, $\alpha_{1, \varepsilon}$ and $\alpha_{2, \varepsilon}$.

points $s_{1, \varepsilon} \in \alpha_{1, \varepsilon}$ and $s_{2, \varepsilon} \in \alpha_{2, \varepsilon}$ so that $T\left(s_{1, \varepsilon}\right), T\left(s_{2, \varepsilon}\right) \in R_{i}\left(\gamma_{\varepsilon}\right)$ for all $0<\varepsilon<\varepsilon_{0}$. By contradictions, if such points do not exist for all $0<\varepsilon<\varepsilon_{0}, T\left(\gamma_{\varepsilon}\right) \subset R_{e}\left(\gamma_{\varepsilon}\right) \cup \gamma_{\varepsilon}$ for all $0<\varepsilon<\varepsilon_{0}$. Therefore, $q$ would be a local repeller, a contradiction. From the existence of these four points, it is clear that the winding number is -1 ; see Figure 6. Finally,

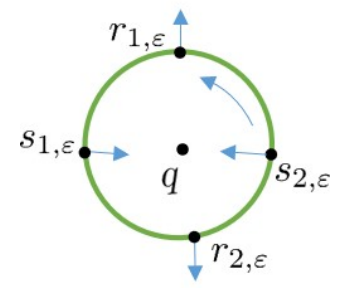

Figure 6: The winding number is -1 .

we observe that $T: \Sigma \mapsto \Sigma$ is an orientation preserving homeomorphism; see [14]. The results follow from properties $\mathrm{C} 2$ ) $-\mathrm{C} 4$ ) of $\Sigma$ and Theorem 2.1 immediately.

Remark 3.2. It is clear that from the proof of Theorem 3.1, one also has index $\left(\left.T\right|_{\Sigma} ^{-1} ; q\right)=$ -1 under the assumptions of Theorem 3.1. In particular, if the map $T$ in Theorem 3.1 has a unique fixed point $q \in \operatorname{Int} \mathbb{R}_{+}^{3}$ with $\operatorname{index}(T ; q)=-1$ and 1 is not an eigenvalue of $D T(q)$, then $D T(q)$ has real eigenvalues $\lambda_{1}, \lambda_{2}, \lambda_{3}$ with $0<\lambda_{1} \leq \lambda_{2}<1<\lambda_{3}$ (see the proof of Theorem 3.1 in [16]), and hence the conclusion of Theorem 3.1 holds. 
Moreover, it follows from [21] that the "Poincaré-Hopf-like" index formula (3) holds for the map $T$ which satisfies the conditions A1)-A3) in Theorem 3.1, and has only finitely many fixed points whose eigenvalues do not equal one. Specifically

$$
\sum_{\theta \in \mathcal{F}_{v}} \operatorname{index}(T ; \theta)+2 \sum_{\theta \in \mathcal{F}_{s}} \operatorname{index}(T ; \theta)+4 \sum_{\theta \in \mathcal{F}_{p}} \operatorname{index}(T ; \theta)=1,
$$

where $\mathcal{F}_{v}\left(\mathcal{F}_{s}, \mathcal{F}_{p}\right)$ denotes the set of all nontrivial axial (planar, positive) fixed points. The condition of $\operatorname{index}(T ; q)=-1$ with $q$ a positive fixed point of $T$ is often difficult to verify directly. Formula (5) simplifies this task since the indices of the fixed points in the boundary are easily computable.

We emphasize that for such maps, uniqueness of fixed point in $\operatorname{Int} \mathbb{R}_{+}^{3}$ and

$$
\sum_{\theta \in \mathcal{F}_{v}} \operatorname{index}(T ; \theta)+2 \sum_{\theta \in \mathcal{F}_{s}} \operatorname{index}(T ; \theta)=5
$$

imply trivial dynamics. Observe that (6) only involves the local behavior of the fixed points of $T$ on the boundary of $\mathbb{R}_{+}^{3}$.

\section{Applications to population models}

In this section we apply the previous results in some classical models that include the Leslie-Gower models, the Atkinson-Allen systems, and the Ricker maps. As emphasized, we solve some open problems suggested in the literature [16, 21, 22, 23], i.e. we show that these systems have trivial dynamics when they possess a unique positive fixed point with index -1 .

\subsection{Leslie-Gower model}

Consider the Leslie-Gower model

$$
T: \mathbb{R}_{+}^{k} \mapsto \mathbb{R}_{+}^{k}, T_{i}(x)=\frac{c_{i} x_{i}}{1+\sum_{j=1}^{k} a_{i j} x_{j}}, c_{i}>1, a_{i j}>0, i, j=1, \cdots, k .
$$

Denote by

$$
\operatorname{CLG}(k):=\left\{T \in \mathcal{X}\left(\mathbb{R}_{+}^{k}\right): T_{i}(x)=\frac{c_{i} x_{i}}{1+\sum_{j=1}^{k} a_{i j} x_{j}}, c_{i}>1, a_{i j}>0, i, j=1, \cdots, k\right\},
$$

where $\mathcal{X}\left(\mathbb{R}_{+}^{k}\right)$ is the set of maps taking $\mathbb{R}_{+}^{k}$ into itself. It was shown in [16] that each map $T \in \mathrm{CLG}(k)$ admits a carrying simplex, say $\Sigma$.

Consider $k=3$. Two maps $T, \hat{T} \in \mathrm{CLG}(3)$ are called equivalent relative to $\partial \Sigma$ if there exists a permutation $\pi$ of $\{1,2,3\}$ such that $T$ has an axial fixed point $s_{\{i\}}$ (resp. a planar fixed point $v_{\{k\}}$ ) if and only if $\hat{T}$ has an axial fixed point $\hat{s}_{\{\pi(i)\}}$ (resp. a planar fixed point $\hat{v}_{\{\pi(k)\}}$ ), and further $s_{\{i\}}$ (resp. $v_{\{k\}}$ ) has the same hyperbolicity and local dynamics as $\hat{s}_{\{\pi(i)\}}$ (resp. $\left.\hat{v}_{\{\pi(k)\}}\right)$. A map $T \in \mathrm{CLG}(3)$ is said to be stable relative to $\partial \Sigma$ if all the fixed points on $\partial \Sigma$ are hyperbolic. We understand that an equivalence class is stable if each mapping in it is stable relative to $\partial \Sigma$; see [16] for more details. 
Note that this equivalence relation only depends on the dynamics of the fixed points on the boundary of $\mathbb{R}_{+}^{3}$, and by the index formula $(6)$, one can obtain the trivial dynamics immediately in some equivalence classes. More precisely, according to [16], there are a total of 33 stable equivalence classes in CLG(3). Moreover, in these classes, $T$ has a unique hyperbolic positive fixed point $q$ with index -1 if and only if $T$ belongs to classes $19-25$ (the precise conditions on the parameters of these classes are given in Table 1).

For systems (7), Theorem 3.1 reads as follows:

Theorem 4.1. Every nontrivial orbit of any map from classes 19 - 25 in CLG(3) converges to some fixed point on $\Sigma$.

Proof. Together with the properties of the carrying simplex $\Sigma$, the result follows from Theorem 3.1 and Remark 3.2 immediately.

Next we provide a further analysis for systems in these classes. According to [16], a map $T \in \mathrm{CLG}(3)$ is in the stable class 19 , if there exists a permutation $\pi$ of $\{1,2,3\}$ after which parameters of $T$ satisfy that

(1) $\alpha_{12}>1, \alpha_{13}>1, \alpha_{21}<1, \alpha_{23}<1, \alpha_{31}<1, \alpha_{32}<1$;

(2) $1+a_{12} \beta_{23}+a_{13} \beta_{32}-c_{1}<0$,

where $\alpha_{i j}=\frac{c_{j} a_{i i}}{a_{j i}\left(c_{i}-1\right)+a_{i i}}, \quad \beta_{i j}=\frac{\left(c_{i}-1\right) a_{j j}-\left(c_{j}-1\right) a_{i j}}{a_{i i} a_{j j}-a_{i j} a_{j i}}$. In class 19, Fix $\left(\left.T\right|_{\Sigma}\right)=$ $\left\{s_{\{1\}}, s_{\{2\}}, s_{\{3\}}, v_{\{1\}}, q\right\}$, where $s_{\{2\}}, s_{\{3\}}$ are hyperbolic local attractors and $s_{\{1\}}, v_{\{1\}}$ are hyperbolic local repellers on $\partial \Sigma$, and $q$ has index -1 .

By using Theorem 4.1, we can derive the whole dynamics on $\Sigma$ for class 19 in CLG(3).

Theorem 4.2. For the stable class 19 in $\mathrm{CLG}(3)$,

(i) $W^{s}\left(q,\left.T\right|_{\Sigma}\right) \cup\left\{s_{\{1\}}, v_{\{1\}}\right\}$ partitions $\Sigma$ into two connected components $\mathcal{A}_{1}$ and $\mathcal{A}_{2}$, where $\mathcal{A}_{1}$ (resp. $\left.\mathcal{A}_{2}\right)$ is the basin of attraction of $s_{\{2\}}$ (resp. $s_{\{3\}}$ ) restricted to $\Sigma$;

(ii) $W^{u}\left(q,\left.T\right|_{\Sigma}\right) \cup\left\{s_{\{2\}}, s_{\{3\}}\right\}$ partitions $\Sigma$ into two connected components $\mathcal{B}_{1}$ and $\mathcal{B}_{2}$, where $\mathcal{B}_{1}$ (resp. $\left.\mathcal{B}_{2}\right)$ is the basin of repulsion of $s_{\{1\}}$ (resp. $v_{\{1\}}$ ) restricted to $\Sigma$;

(iii) $W^{s}\left(q,\left.T\right|_{\Sigma}\right) \cup W^{u}\left(q,\left.T\right|_{\Sigma}\right) \cup\left\{s_{\{1\}}, s_{\{2\}}, s_{\{3\}}, v_{\{1\}}\right\}$ partitions $\Sigma$ into four invariant components.

Proof. It follows from the proof of Theorem 3.1 that index $\left(\left.T\right|_{\Sigma} ; q\right)=-1$. Note that $F i x\left(\left.T\right|_{\Sigma}\right) \cap \partial \Sigma=\left\{s_{\{1\}}, s_{\{2\}}, s_{\{3\}}, v_{\{1\}}\right\}$, of which $s_{\{2\}}, s_{\{3\}}$ are local attractors and $s_{\{1\}}, v_{\{1\}}$ are local repellers for $\left.T\right|_{\Sigma}$. Therefore, the conclusions of Theorem 2.4 hold for $\left.T\right|_{\Sigma}: \Sigma \mapsto \Sigma$. Now the conclusions (i)-(iii) are immediate.

We can argue in the same manner with the rest of classes.

Theorem 4.3. For classes $20-25$ in CLG(3), each map has two local attractors and repellers on $\partial \Sigma$, and the stable (resp. unstable) manifold $W^{s}\left(q,\left.T\right|_{\Sigma}\right)\left(\operatorname{resp} . W^{u}\left(q,\left.T\right|_{\Sigma}\right)\right.$ ) together with the two repellers (resp. attractors) partitions $\Sigma$ into two connected components. Furthermore, $W^{s}\left(q,\left.T\right|_{\Sigma}\right)$ and $W^{u}\left(q,\left.T\right|_{\Sigma}\right)$ together with the two attractors and repellers partition $\Sigma$ into four invariant components. 


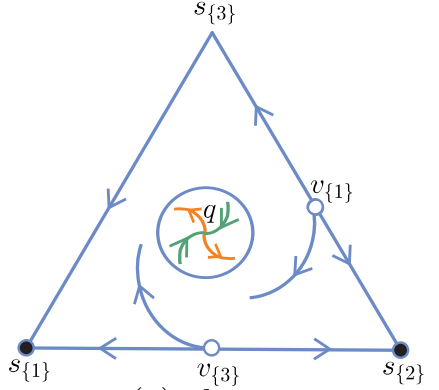

(a) class 20

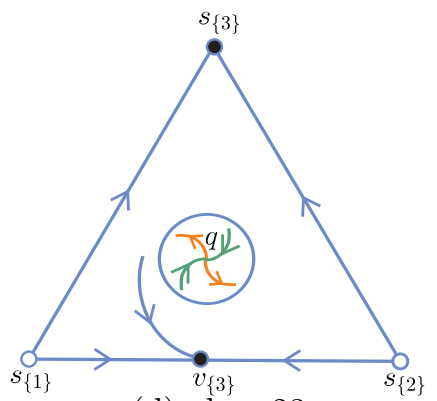

(d) class 23

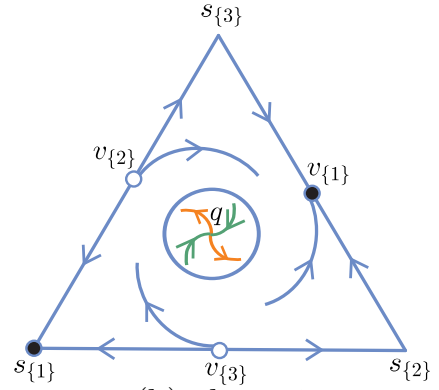

(b) class 21

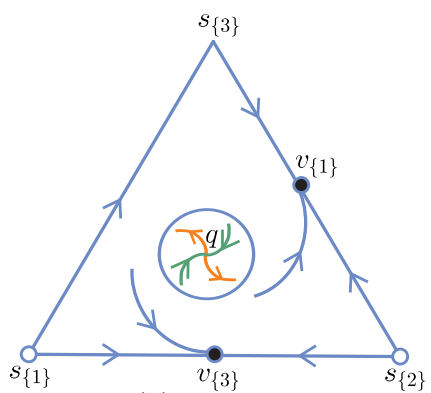

(e) class 24

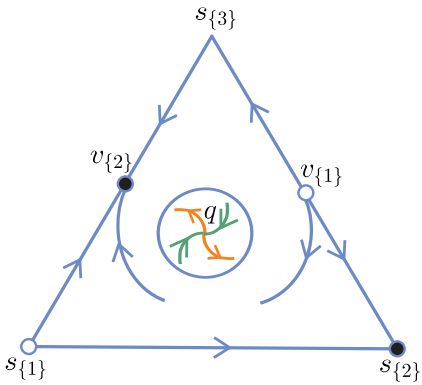

(c) class 22

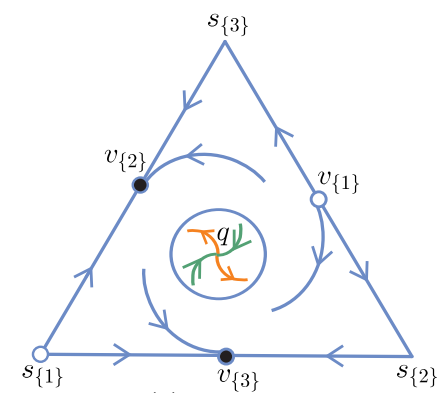

(f) class 25

Figure 7: The dynamics on $\Sigma$ for classes $20-25$ in CLG(3). Redrawn from [16]. The fixed point notation is as in Figure 1 .

Proof. According to [16], the dynamics (partial) on $\Sigma$ for classes $20-25$ is as shown in Figure 7 .

We first show the conclusions for class 20. By the dynamics shown in Figure 7(a), the fixed point $s_{\{3\}}$ is a saddle whose one branch of the stable manifold and one branch of unstable manifold are contained in $\partial \Sigma$, i.e., $\widehat{s_{\{3\}} s_{\{1\}}}$ (resp. $\widehat{s_{\{3\}} v_{\{1\}}}$ ) in Figure $7($ a) is the branch of unstable (resp. stable) manifold contained in $\partial \Sigma$, where $\widehat{s\{3\} S\{1\}}$ (resp. $\widehat{s_{\{3\}} v_{\{1\}}}$ ) is the open arc on $\partial \Sigma$ joining $s_{\{1\}}$ and $s_{\{3\}}$ (resp. $v_{\{1\}}$ and $s_{\{3\}}$ ); $s_{\{1\}}, s_{\{2\}}$ are two local attractors, and $v_{\{1\}}, v_{\{3\}}$ are two local repellers for $\left.T\right|_{\Sigma}$. Then by similar arguments in Theorem 2.4, we have Int $\Sigma=W^{s}\left(q,\left.T\right|_{\Sigma}\right) \cup\left(\mathcal{C}_{1} \cup \mathcal{C}_{2}\right) \cap \operatorname{Int} \Sigma$, and $\operatorname{Int} \Sigma=W^{u}\left(q,\left.T\right|_{\Sigma}\right) \cup\left(\mathcal{C}_{3} \cup \mathcal{C}_{4}\right) \cap \operatorname{Int} \Sigma$, where $\mathcal{C}_{1}$ (resp. $\left.\mathcal{C}_{2}\right)$ is the basin of attraction of $s_{\{1\}}$ (resp. $\left.s_{\{2\}}\right)$ and $\mathcal{C}_{3}$ (resp. $\mathcal{C}_{4}$ ) is the basin of repulsion of $v_{\{1\}}$ (resp. $v_{\{3\}}$ ). Moreover, $W^{s}\left(q,\left.T\right|_{\Sigma}\right) \cup W^{u}\left(q,\left.T\right|_{\Sigma}\right) \cup\left\{s_{\{1\}}, s_{\{2\}}, v_{\{1\}}, v_{\{3\}}\right\}$ partitions $\Sigma$ into four invariant components.

The conclusions for classes 21-25 can be obtained similarly as the analysis of class 20 .

The parameter conditions for each of classes $19-25$ are listed in Table 1. Our results have a topological flavour and do not involve hyperbolicity conditions. Since the unstable manifold of $q$ for classes $19-25$ is uniquely defined which is contained in the carrying simplex, we know that $W^{u}\left(q,\left.T\right|_{\Sigma}\right)$ is a simple curve. But we emphasize that Theorems 4.2 and 4.3 does not guarantee that $W^{s}\left(q,\left.T\right|_{\Sigma}\right)$ is a simple curve. 
Table 1: Stable equivalence classes $19-25$ in CLG(3), where

$\alpha_{i j}=\frac{c_{j} a_{i i}}{a_{j i}\left(c_{i}-1\right)+a_{i i}}, \quad \beta_{i j}=\frac{\left(c_{i}-1\right) a_{j j}-\left(c_{j}-1\right) a_{i j}}{a_{i i} a_{j j}-a_{i j} a_{j i}}$

for $i, j=1,2,3$ and $i \neq j$, and $\Sigma$ with the corresponding conditions in each class is given by a representative element in that class, i.e. there exists a permutation $\pi$ of $\{1,2,3\}$ after which parameters of the map satisfy the corresponding inequalities in that class. The parameter conditions are obtained from [16]. Each map in these classes has trivial dynamics.

\begin{tabular}{|c|c|c|}
\hline Class & & he Corresponding Parameter Conditions \\
\hline 19 & $\begin{array}{l}\text { (i) } \\
\text { (ii) }\end{array}$ & $\begin{array}{l}\alpha_{12}>1, \alpha_{13}>1, \alpha_{21}<1, \alpha_{23}<1, \alpha_{31}<1, \alpha_{32}<1 \\
1+a_{12} \beta_{23}+a_{13} \beta_{32}-c_{1}<0\end{array}$ \\
\hline 20 & $\begin{array}{l}\text { (i) } \\
\text { (ii) } \\
\text { (iii) }\end{array}$ & $\begin{array}{l}\alpha_{12}<1, \alpha_{13}<1, \alpha_{21}<1, \alpha_{23}<1, \alpha_{31}>1, \alpha_{32}<1 \\
1+a_{12} \beta_{23}+a_{13} \beta_{32}-c_{1}<0 \\
1+a_{31} \beta_{12}+a_{32} \beta_{21}-c_{3}<0\end{array}$ \\
\hline 21 & $\begin{array}{l}\text { (i) } \\
\text { (ii) } \\
\text { (iii) } \\
\text { (iv) }\end{array}$ & $\begin{array}{l}\alpha_{12}<1, \alpha_{13}<1, \alpha_{21}<1, \alpha_{23}>1, \alpha_{31}<1, \alpha_{32}>1 \\
1+a_{12} \beta_{23}+a_{13} \beta_{32}-c_{1}>0 \\
1+a_{21} \beta_{13}+a_{23} \beta_{31}-c_{2}<0 \\
1+a_{31} \beta_{12}+a_{32} \beta_{21}-c_{3}<0\end{array}$ \\
\hline 22 & $\begin{array}{l}\text { (i) } \\
\text { (ii) } \\
\text { (iii) }\end{array}$ & $\begin{array}{l}\alpha_{12}>1, \alpha_{13}>1, \alpha_{21}<1, \alpha_{23}<1, \alpha_{31}>1, \alpha_{32}<1 \\
1+a_{12} \beta_{23}+a_{13} \beta_{32}-c_{1}<0 \\
1+a_{21} \beta_{13}+a_{23} \beta_{31}-c_{2}>0\end{array}$ \\
\hline 23 & $\begin{array}{l}\text { (i) } \\
\text { (ii) }\end{array}$ & $\begin{array}{l}\alpha_{12}>1, \alpha_{13}>1, \alpha_{21}>1, \alpha_{23}>1, \alpha_{31}<1, \alpha_{32}<1 \\
1+a_{31} \beta_{12}+a_{32} \beta_{21}-c_{3}>0\end{array}$ \\
\hline 24 & $\begin{array}{l}\text { (i) } \\
\text { (ii) } \\
\text { (iii) }\end{array}$ & $\begin{array}{l}\alpha_{12}>1, \alpha_{13}>1, \alpha_{21}>1, \alpha_{23}>1, \alpha_{31}<1, \alpha_{32}>1 \\
1+a_{12} \beta_{23}+a_{13} \beta_{32}-c_{1}>0 \\
1+a_{31} \beta_{12}+a_{32} \beta_{21}-c_{3}>0\end{array}$ \\
\hline 25 & $\begin{array}{l}\text { (i) } \\
\text { (ii) } \\
\text { (iii) } \\
\text { (iv) }\end{array}$ & $\begin{array}{l}\alpha_{12}>1, \alpha_{13}>1, \alpha_{21}>1, \alpha_{23}<1, \alpha_{31}>1, \alpha_{32}<1 \\
1+a_{12} \beta_{23}+a_{13} \beta_{32}-c_{1}<0 \\
1+a_{21} \beta_{13}+a_{23} \beta_{31}-c_{2}>0 \\
1+a_{31} \beta_{12}+a_{32} \beta_{21}-c_{3}>0\end{array}$ \\
\hline
\end{tabular}




\subsection{Atkinson-Allen system}

Consider the generalized Atkinson-Allen model $T: \mathbb{R}_{+}^{k} \mapsto \mathbb{R}_{+}^{k}$ given by

$$
T_{i}(x)=\frac{\left(1+r_{i}\right)\left(1-c_{i}\right) x_{i}}{1+\sum_{j=1}^{k} a_{i j} x_{j}}+c_{i} x_{i}, 0<c_{i}<1, a_{i j}, r_{i}>0, i, j=1, \cdots, k .
$$

When $r_{i}=1$ and $c_{i}=c$, the model (8) reduces to the standard Atkinson-Allen model [21]

$$
T_{i}(x)=\frac{2(1-c) x_{i}}{1+\sum_{j=1}^{k} a_{i j} x_{j}}+c x_{i}, 0<c<1, a_{i j}>0, i, j=1, \ldots, k .
$$

Let $K=\{1, \ldots, k\}$. Denote by

$$
\operatorname{CGAA}(k):=\left\{T \in \mathcal{X}\left(\mathbb{R}_{+}^{k}\right): T_{i}(x)=\frac{\left(1+r_{i}\right)\left(1-c_{i}\right) x_{i}}{1+\sum_{j=1}^{k} a_{i j} x_{j}}+c_{i} x_{i}, 0<c_{i}<1, a_{i j}, r_{i}>0, i, j \in K\right\}
$$

the Atkinson-Allen type competitive map set on $\mathbb{R}_{+}^{k}$. It is shown in [22] that each $T \in \operatorname{CGAA}(k)$ admits a carrying simplex. For CGAA(3), Gyllenberg et al. [22] defined a similar equivalence relation as CLG(3), named equivalent relative to $\partial \Sigma$. They showed that there are a total of 33 stable equivalence classes in CGAA(3). Moreover, in these classes, $T$ has a unique hyperbolic positive fixed point with index -1 if and only if $T$ belongs to classes $19-25$, and see Table 2 for the precise parameter conditions on each of these classes.

For systems (8), Theorem 3.1 reads as follows:

Theorem 4.4. Every nontrivial orbit of any map from classes $19-25$ in CGAA(3) converges to some fixed point on $\Sigma$.

Note that, systems (8) in each of classes $19-25$ for CGAA(3) have the similar partial dynamics shown in Figure 1 and Figure 7 as those systems in CLG(3); see [22]. So, the following results on trivial dynamics for CGAA(3) can be obtained immediately just as the analysis for CLG(3) by using Theorems 2.4 and 4.4 .

Theorem 4.5. The conclusions of Theorems 4.2 and 4.3 hold for classes $19-25$ in CGAA(3). 
Table 2: Stable equivalence classes $19-25$ in CGAA(3), where

$\gamma_{i j}:=r_{j}-a_{j i} \frac{r_{i}}{a_{i i}}, \quad \beta_{i j}=\frac{r_{i} a_{j j}-r_{j} a_{i j}}{a_{i i} a_{j j}-a_{i j} a_{j i}}$

for $i, j=1,2,3$ and $i \neq j$, and $\Sigma$ with the corresponding conditions in each class is given by a representative element in that class, i.e. there exists a permutation $\pi$ of $\{1,2,3\}$ after which parameters of the map satisfy the corresponding inequalities in that class. The parameter conditions are obtained from [22]. Each map in these classes has trivial dynamics.

\begin{tabular}{|c|c|c|}
\hline Class & & The Corresponding Parameter Conditions \\
\hline 19 & $\begin{array}{l}\text { (i) } \\
\text { (ii) }\end{array}$ & $\begin{array}{l}\gamma_{12}>0, \gamma_{13}>0, \gamma_{21}<0, \gamma_{23}<0, \gamma_{31}<0, \gamma_{32}<0 \\
a_{12} \beta_{23}+a_{13} \beta_{32}-r_{1}<0\end{array}$ \\
\hline 20 & $\begin{array}{l}\text { (i) } \\
\text { (ii) } \\
\text { (iii) }\end{array}$ & $\begin{array}{l}\gamma_{12}<0, \gamma_{13}<0, \gamma_{21}<0, \gamma_{23}<0, \gamma_{31}>0, \gamma_{32}<0 \\
a_{12} \beta_{23}+a_{13} \beta_{32}-r_{1}<0 \\
a_{31} \beta_{12}+a_{32} \beta_{21}-r_{3}<0\end{array}$ \\
\hline 21 & $\begin{array}{l}\text { (i) } \\
\text { (ii) } \\
\text { (iii) } \\
\text { (iv) }\end{array}$ & $\begin{array}{l}\gamma_{12}<0, \gamma_{13}<0, \gamma_{21}<0, \gamma_{23}>0, \gamma_{31}<0, \gamma_{32}>0 \\
a_{12} \beta_{23}+a_{13} \beta_{32}-r_{1}>0 \\
a_{21} \beta_{13}+a_{23} \beta_{31}-r_{2}<0 \\
a_{31} \beta_{12}+a_{32} \beta_{21}-r_{3}<0\end{array}$ \\
\hline 22 & $\begin{array}{l}\text { (i) } \\
\text { (ii) } \\
\text { (iii) }\end{array}$ & $\begin{array}{l}\gamma_{12}>0, \gamma_{13}>0, \gamma_{21}<0, \gamma_{23}<0, \gamma_{31}>0, \gamma_{32}<0 \\
a_{12} \beta_{23}+a_{13} \beta_{32}-r_{1}<0 \\
a_{21} \beta_{13}+a_{23} \beta_{31}-r_{2}>0\end{array}$ \\
\hline 23 & $\begin{array}{l}\text { (i) } \\
\text { (ii) }\end{array}$ & $\begin{array}{l}\gamma_{12}>0, \gamma_{13}>0, \gamma_{21}>0, \gamma_{23}>0, \gamma_{31}<0, \gamma_{32}<0 \\
a_{31} \beta_{12}+a_{32} \beta_{21}-r_{3}>0\end{array}$ \\
\hline 24 & $\begin{array}{l}\text { (i) } \\
\text { (ii) } \\
\text { (iii) }\end{array}$ & $\begin{array}{l}\gamma_{12}>0, \gamma_{13}>0, \gamma_{21}>0, \gamma_{23}>0, \gamma_{31}<0, \gamma_{32}>0 \\
a_{12} \beta_{23}+a_{13} \beta_{32}-r_{1}>0 \\
a_{31} \beta_{12}+a_{32} \beta_{21}-r_{3}>0\end{array}$ \\
\hline 25 & $\begin{array}{l}\text { (i) } \\
\text { (ii) } \\
\text { (iii) } \\
\text { (iv) }\end{array}$ & $\begin{array}{l}\gamma_{12}>0, \gamma_{13}>0, \gamma_{21}>0, \gamma_{23}<0, \gamma_{31}>0, \gamma_{32}<0 \\
a_{12} \beta_{23}+a_{13} \beta_{32}-r_{1}<0 \\
a_{21} \beta_{13}+a_{23} \beta_{31}-r_{2}>0 \\
a_{31} \beta_{12}+a_{32} \beta_{21}-r_{3}>0\end{array}$ \\
\hline
\end{tabular}


Remark 4.6. Since the standard Atkinson-Allen model (9) is a special case of the generalized Atkinson-Allen model (8), the conclusions of Theorems 4.4 and 4.5 hold naturally for classes $19-25$ of 3D standard Atkinson-Allen models (9) in [21], i.e. the classes $19-25$ of 3D standard Atkinson-Allen models have trivial dynamics.

\subsection{Ricker map}

Consider the Ricker map

$$
T: \mathbb{R}_{+}^{k} \mapsto \mathbb{R}_{+}^{k}, T_{i}(x)=x_{i} \exp \left(c_{i}\left(1-\sum_{j=1}^{k} a_{i j} x_{j}\right)\right), c_{i}, a_{i j}>0, i, j=1, \cdots, k .
$$

It is shown in [23] that the Ricker map (10) admits a carrying simplex if the parameters satisfy

$$
c_{i}<a_{i i} / \sum_{j=1}^{k} a_{i j}, \text { or } c_{i}<1 /\left(\sum_{j=1}^{k} \frac{a_{i j}}{a_{j j}}\right), i=1, \cdots, k .
$$

Denote by

$$
\operatorname{CRC}(k):=\left\{T \in \mathcal{X}\left(\mathbb{R}_{+}^{k}\right): T_{i}(x)=x_{i} \exp \left(c_{i}\left(1-\sum_{j=1}^{k} a_{i j} x_{j}\right)\right), c_{i}, a_{i j}>0,111 \text { holds }\right\}
$$

the subset of the Ricker type competitive map set on $\mathbb{R}_{+}^{k}$ such that each one admits a carrying simplex.

For $\mathrm{CRC}(3)$, a similar equivalence relation as $\mathrm{CLG}(3)$ and $\mathrm{CAA}(3)$, i.e. equivalent relative to $\partial \Sigma$, was also defined by Gyllenberg et al. [23]. They showed that there are a total of 33 stable equivalence classes in CRC(3). Moreover, in these classes, $T$ has a unique hyperbolic positive fixed point with index -1 if and only if $T$ belongs to classes $19-25$, and the precise parameter conditions on each of these classes are listed in Table 3.

It is easy to check that for each map in $\mathrm{CRC}(3)$, it satisfies conditions A1)-A3) in Theorem 3.1. For systems (10), Theorem 3.1 reads as follows:

Theorem 4.7. Every nontrivial orbit of any map from classes $19-25$ in $\mathrm{CRC}(3)$ converges to some fixed point on $\Sigma$.

Note also that, systems (10) in each of classes $19-25$ for CRC(3) have the similar partial dynamics shown in Figure 1 and Figure 7 as those systems in CLG(3); see

[23. So, the following results on trivial dynamics for $\mathrm{CRC}(3)$ can also be obtained immediately just as the analysis for CLG(3) by using Theorems 2.4 and 4.7 .

Theorem 4.8. The conclusions in Theorems 4.2 and 4.3 hold for classes $19-25$ in $\mathrm{CRC}(3)$. 
Table 3: Stable equivalence classes $19-25$ in $\mathrm{CRC}(3)$, where $\alpha_{i j}:=a_{i i}-a_{j i}, \quad \beta_{i j}=\frac{a_{j j}-a_{i j}}{a_{i i} a_{j j}-a_{i j} a_{j i}}$

for $i, j=1,2,3$ and $i \neq j$. The $\Sigma$ with corresponding parameters in each class is given by a representative element in that class, i.e. there exists a permutation $\pi$ of $\{1,2,3\}$ after which parameters of the map satisfy the corresponding inequalities in that class (in addition to the parameter conditions listed for each class, the parameters also satisfy the common additional condition (11)). The parameter conditions are obtained from 23. Each map in these classes has trivial dynamics.

\begin{tabular}{|c|c|c|}
\hline Class & & he Corresponding Parameter Conditions \\
\hline 19 & $\begin{array}{l}\text { (i) } \\
\text { (ii) }\end{array}$ & $\begin{array}{l}\alpha_{12}>0, \alpha_{13}>0, \alpha_{21}<0, \alpha_{23}<0, \alpha_{31}<0, \alpha_{32}<0 \\
a_{12} \beta_{23}+a_{13} \beta_{32}<1\end{array}$ \\
\hline 20 & $\begin{array}{l}\text { (i) } \\
\text { (ii) } \\
\text { (iii) }\end{array}$ & $\begin{array}{l}\alpha_{12}<0, \alpha_{13}<0, \alpha_{21}<0, \alpha_{23}<0, \alpha_{31}>0, \alpha_{32}<0 \\
a_{12} \beta_{23}+a_{13} \beta_{32}<1 \\
a_{31} \beta_{12}+a_{32} \beta_{21}<1\end{array}$ \\
\hline 21 & $\begin{array}{l}\text { (i) } \\
\text { (ii) } \\
\text { (iii) } \\
\text { (iv) }\end{array}$ & $\begin{array}{l}\alpha_{12}<0, \alpha_{13}<0, \alpha_{21}<0, \alpha_{23}>0, \alpha_{31}<0, \alpha_{32}>0 \\
a_{12} \beta_{23}+a_{13} \beta_{32}>1 \\
a_{21} \beta_{13}+a_{23} \beta_{31}<1 \\
a_{31} \beta_{12}+a_{32} \beta_{21}<1\end{array}$ \\
\hline 22 & $\begin{array}{l}\text { (i) } \\
\text { (ii) } \\
\text { (iii) }\end{array}$ & $\begin{array}{l}\alpha_{12}>0, \alpha_{13}>0, \alpha_{21}<0, \alpha_{23}<0, \alpha_{31}>0, \alpha_{32}<0 \\
a_{12} \beta_{23}+a_{13} \beta_{32}<1 \\
a_{21} \beta_{13}+a_{23} \beta_{31}>1\end{array}$ \\
\hline 23 & $\begin{array}{l}\text { (i) } \\
\text { (ii) }\end{array}$ & $\begin{array}{l}\alpha_{12}>0, \alpha_{13}>0, \alpha_{21}>0, \alpha_{23}>0, \alpha_{31}<0, \alpha_{32}<0 \\
a_{31} \beta_{12}+a_{32} \beta_{21}>1\end{array}$ \\
\hline 24 & $\begin{array}{l}\text { (i) } \\
\text { (ii) } \\
\text { (iii) }\end{array}$ & $\begin{array}{l}\alpha_{12}>0, \alpha_{13}>0, \alpha_{21}>0, \alpha_{23}>0, \alpha_{31}<0, \alpha_{32}>0 \\
a_{12} \beta_{23}+a_{13} \beta_{32}>1 \\
a_{31} \beta_{12}+a_{32} \beta_{21}>1\end{array}$ \\
\hline 25 & $\begin{array}{l}\text { (i) } \\
\text { (ii) } \\
\text { (iii) } \\
\text { (iv) }\end{array}$ & $\begin{array}{l}\alpha_{12}>0, \alpha_{13}>0, \alpha_{21}>0, \alpha_{23}<0, \alpha_{31}>0, \alpha_{32}<0 \\
a_{12} \beta_{23}+a_{13} \beta_{32}<1 \\
a_{21} \beta_{13}+a_{23} \beta_{31}>1 \\
a_{31} \beta_{12}+a_{32} \beta_{21}>1\end{array}$ \\
\hline
\end{tabular}




\section{Conclusions}

This paper shows that an orientation preserving homeomorphism $f$ from a topological disk $D$ onto itself with a unique fixed point of index -1 in $\operatorname{Int} D$ has trivial dynamics, i.e., every orbit converges to some fixed point (Theorem 2.1), and the whole dynamics on the disk can be described further (Theorem 2.4). The result on the trivial dynamics is

extended to the 3D discrete-time Kolmogorov systems on $\mathbb{R}_{+}^{3}$ induced by (2) admitting a carrying simplex (Theorem 3.1). Moreover, due to the "Poincaré-Hopf-like" index formula (3), the dynamical behavior can be sometimes determined completely by the number of fixed points on the boundary and the local behavior around them when there is a unique positive fixed point (Remark 3.2). The results are applied to the Leslie-Gower models, the Atkinson-Allen models, and the Ricker models. Specifically, these models (3D) do have trivial dynamics when they admit a unique interior fixed point with index -1 , and hence the open problems on class 19-25 for these systems in [16, 21, 22, 23] are solved.

\section{Acknowledgement}

The authors are greatly indebted to an anonymous referee for very careful reading, pointing out some errors in the original manuscript and providing lots of other very inspiring and helpful comments and suggestions which led to much improvement of the earlier version of this paper. This work is supported by the Academy of Finland and the Spanish project MTM2017-87697P.

\section{References}

[1] M W Hirsch. Systems of differential equations which are competitive or cooperative. III: Competing species. Nonlinearity, 1:51-71, 1988.

[2] R Ortega and L Á Sánchez. Abstract competitive systems and orbital stability in $\mathbb{R}^{3}$. Proc. Amer. Math. Soc., 128:2911-2919, 2000.

[3] H R Zhu and H L Smith. Stable periodic orbits for a class of three dimensional competitive systems. J. Differential Equations, 110:143-156, 1994.

[4] M L Zeeman. Hopf bifurcations in competitive three-dimensional Lotka-Volterra systems. Dynam. Stability Systems, 8:189-217, 1993.

[5] $\mathrm{P}$ van den Driessche and M L Zeeman. Three-dimensional competitive Lotka-Volterra systems with no periodic orbits. SIAM J. Appl. Math, 58:227-234, 1998.

[6] X Chen, J Jiang, and L Niu. On Lotka-Volterra equations with identical minimal intrinsic growth rate. SIAM J. Appl. Dyn. Syst., 14:1558-1599, 2015.

[7] E C Zeeman and M L Zeeman. From local to global behavior in competitive Lotka-Volterra systems. Trans. Amer. Math. Soc., 355:713-734, 2002.

[8] E C Zeeman and M L Zeeman. An n-dimensional competitive Lotka-Volterra system is generically determined by the edges of its carrying simplex. Nonlinearity, 15:2019-2032, 2002.

[9] H L Smith. Periodic competitive differential equations and the discrete dynamics of competitive maps. J. Differential Equations, 64:165-194, 1986. 
[10] Y Wang and J Jiang. Uniqueness and attractivity of the carrying simplex for discrete-time competitive dynamical systems. J. Differential Equations, 186:611-632, 2002.

[11] M W Hirsch. On existence and uniqueness of the carrying simplex for competitive dynamical systems. J. Biol. Dyn., 2:169-179, 2008.

[12] O Diekmann, Y Wang, and P Yan. Carrying simplices in discrete competitive systems and agestructured semelparous populations. Discrete Contin. Dyn. Syst., 20:37-52, 2008.

[13] J Jiang and J Mierczyński and Y Wang. Smoothness of the carrying simplex for discretetime competitive dynamical systems: A characterization of neat embedding. J. Differential Equations, 246: 1623-1672, 2009.

[14] A Ruiz-Herrera. Exclusion and dominance in discrete population models via the carrying simplex. J. Difference Equ. Appl., 19:96-113, 2013.

[15] J Jiang, L Niu, and Y Wang. On heteroclinic cycles of competitive maps via carrying simplices. J. Math. Biol., 72:939-972, 2016.

[16] J Jiang and L Niu. On the equivalent classification of three-dimensional competitive Leslie/Gower models via the boundary dynamics on the carrying simplex. J. Math. Biol., 74:1223-1261, 2017.

[17] E C Balreira, S Elaydi, and R Luís. Global stability of higher dimensional monotone maps. $J$. Diff. Equ. Appl., 23:2037-2071, 2017.

[18] J Mierczyński. The $C^{1}$ property of convex carrying simplices for three-dimensional competitive maps. J. Diff. Equ. Appl., January, 2018.

[19] M Hénon. A two-dimensional mapping with a strange attractor. Comm. Math. Phys., 50:69-77, 1976.

[20] R M May and G F Oster. Bifurcations and dynamic complexity in simple ecological models. Am. Nat., 110:573-599, 1976.

[21] J Jiang and L Niu. On the equivalent classification of three-dimensional competitive Atkinson/Allen models relative to the boundary fixed points. Discrete Contin. Dyn. Syst., $36: 217-244,2016$.

[22] M Gyllenberg and J Jiang and L Niu and P Yan. On the classification of generalized competitive Atkinson-Allen models via the dynamics on the boundary of the carrying simplex. Discrete Contin. Dyn. Syst., 38: 615-650, 2018.

[23] M Gyllenberg, J Jiang, L Niu, and P Yan. On the dynamics of multi-species Ricker models admitting a carrying simplex. Submitted.

[24] J Jiang, L Niu, and D Zhu. On the complete classification of nullcline stable competitive threedimensional Gompertz models. Nonlinear Anal. Real World Appl., 20:21-35, 2014.

[25] E N Dancer. Degree Theory on Convex Sets and Applications to Bifurcation, in: Calculus of Variations and Partial Differential Equations. Pisa 1996, Springer-Verlag, 2000.

[26] A Dold. Fixed point index and fixed point theorem for Euclidean neighborhood retracts. Topology, 4:1-8, 1965.

[27] L E J Brouwer. Beweis des ebenen translationssatzes. Math. Ann., 72:37-54, 1912.

[28] M Brown. A new proof of Brouwer's lemma on translation arcs. Houston J. Math., 10:35-41, 1984.

[29] G Graff and A Ruiz-Herrera. A strategy to locate fixed points and global perturbations of ODE's: mixing topology with metric conditions. J. Dynam. Differential Equations, 26:93-107, 2014.

[30] R Ortega. Topology of the plane and periodic differential equations. www.ugr.es/local/ecuadif/fuentenueva.htm.

[31] J P LaSalle. The Stability of Dynamical Systems, volume 25. SIAM, 1976. 